\title{
Matem-SRB: resultados de su primer año de implementación
}

\section{Matem-SRB: Results of Its First Year of Implementation}

\author{
Johan Espinoza González \\ Universidad Nacional \\ Pérez Zeledón, Costa Rica \\ jespinoza@una.cr \\ Fabián Hernández Vargas \\ Universidad Nacional \\ Pérez Zeledón, Costa Rica \\ fabian.hernandez.vargas@una.ac.cr
}

Recibido:18/09/2020 - Aceptado: 20/01/2021

Resumen. Matem-SRB es un proyecto que combina la extensión, la docencia y la investigación a partir del principio de regionalización y acción social. Les brinda a los y las docentes de Matemática la oportunidad de capacitarse en distintos temas y al estudiantado de secundaria la oportunidad de llevar una educación matemática integral, situación que les ayuda a incrementar sus niveles de conocimiento en la disciplina, teniendo la oportunidad de relacionarse con el ambiente universitario. Matem-SRB integra la participación de distintos actores, como la Sede Regional Brunca, la Escuela de Matemática de la UNA, las instituciones participantes y los profesores y las profesoras que imparten tutorías, situación que promueve una colaboración constante en busca de brindarles a los y las discentes que se encuentran en algunas de las zonas con menor desarrollo del país nuevas oportunidades para su crecimiento académico y personal.

Palabras clave: Matemática, enseñanza, región sur.

Abstract. MATEM-SRB is a project that combines extension, teaching, and research; it is based on the principle of regionalization and social action. It offers Mathematics teachers the opportunity to train in different subjects; it also allows secondary school students the opportunity to receive a comprehensive mathematics education, helping them increase their knowledge levels in the discipline and allowing them to 
Revista Universidad en Diálogo • Vol. 11, N. 2 2, Julio-Diciembre, 2021 • 31-43

ISSN 2215-2849 • EISSN: 2215-4752

URL: http://www.revistas.una.ac.cr/index.php/dialogo/index CorReo ElECTRÓNICO: universidadendialogo@una.cr DOI: http://doi.org/10.15359/udre.11-2.2

interact with the university environment. MATEM-SRB integrates the participation of different actors, such as Universidad Nacional de Costa Rica (UNA), Brunca Regional Campus, the UNA School of Mathematics, participating institutions, and tutors. This situation promotes constant collaboration in search of providing students living in some country's least developed areas with new opportunities for academic and personal growth.

Keywords: Mathematics, teaching, southern region.

\section{Introducción}

En Costa Rica, la enseñanza y el aprendizaje de la Matemática han sido un reto que las instituciones involucradas en estos procesos deben asumir mediante la implementación de acciones que incrementen el rendimiento académico en esta asignatura.

Asimismo, las estadísticas en relación con las Pruebas Nacionales de Bachillerato de Matemática, la prueba PISA y los datos con que cuentan las universidades estatales sobre los cursos de Matemática no son alentadores. La Región Brunca no está exenta de estos problemas, por lo que es necesaria la implementación de proyectos que colaboren al mejoramiento de la educación y del aprendizaje de la Matemática.

Es así como las universidades públicas de Costa Rica desarrollan proyectos que brindan apoyo a la educación matemática. Tal es el caso del proyecto Matemática para la Enseñanza Media (Matem), desarrollado en la Universidad Nacional (UNA), la Universidad de Costa Rica (UCR) y el Tecnológico de Costa Rica (TEC), y que por muchos años ha fortalecido el aprendizaje de la disciplina en distintas regiones del país. Sin embargo, este esfuerzo no ha generado un gran impacto en la zona sur del país, ya que por varios años han sido pocos los colegios de la región que han participado en alguna de sus modalidades, debido a factores geográficos, económicos, sociales o culturales.

A partir de esta situación y en busca de una regionalización de la extensión y la acción social es que la Sede Regional Brunca y la Escuela de Matemática de la Universidad Nacional, en convenio, plantean la implementación del proyecto Matem Sede Regional Brunca (Matem-SRB), con código SIA 0266-18, durante el periodo 2019-2021. Esta iniciativa busca convertirse en un canal de comunicación que fomente y propicie la participación de las instituciones de educación secundaria en el proyecto, dando prioridad a aquellas instituciones que se encuentren ubicadas en las zonas de menor desarrollo de la región. 
URL: http://www.revistas.una.ac.cr/index.php/dialogo/index

CORREO ELECTRÓNICO: universidadendialogo@una.cr

DOI: http://doi.org/10.15359/udre.11-2.2

\section{Desarrollo de contenidos}

\section{Matem-SRB y la realidad del país}

Costa Rica presenta una importante necesidad de formación de profesionales en carreras técnicas y en ingenierías, debido a la instalación de empresas de alta tecnología que contribuyen al desarrollo económico y productivo del país. Esto conlleva el desarrollo del área científica, que requiere la formación de distintas áreas del conocimiento, como la matemática.

De esta forma, para incrementar la competitividad del país, se requiere un impulso cada vez mayor de la educación superior y técnica, eficiencia en el mercado de bienes, buen funcionamiento del mercado de trabajo, mercados financieros desarrollados y la habilidad de aprovechar las tecnologías ya existentes. Este escenario de productividad demanda que los países sean capaces de competir con productos nuevos y únicos, utilizando procesos de producción innovadores y sofisticados como medio para alcanzar altos niveles de desarrollo humano (Estado de la Nación, 2014).

Ante este escenario, el proyecto se enfoca en estudiantes que cursan los últimos niveles de secundaria, ya que una serie de indicadores muestran que el panorama del rendimiento en esta área de los y las estudiantes que pronto ingresarán a la universidad no es para nada positivo (Estado de la Nación, 2017).

En este sentido, el análisis estadístico de los ítems de las Pruebas de Bachillerato de Matemática en el 2017 evidencia notoriamente que la población muestra debilidades en la resolución de problemas en sus diferentes áreas y el manejo de elementos básicos.

Asimismo, el Programa para la Evaluación Internacional de Estudiantes, por sus siglas en inglés PISA, ubica a Costa Rica en el año 2018 en el área de la matemática (último año de su aplicación) en el puesto 63 de los 79 países evaluados. Situación que además debe convertirse en un factor de preocupación, ya que el país bajó de puesto en relación con la evaluación del 2015 (Estado de la Nación, 2019).

A nivel de las universidades públicas es posible también encontrar indicadores que muestran que los conocimientos básicos de Matemática que poseen los y las estudiantes al ingresar a la educación superior son deficientes. Esto se puede visualizar en los niveles de aprobación que se dan en los cursos de Matemática que se imparten en las distintas carreras, e incluso en los resultados de los exámenes de diagnóstico de Matemática que realiza la Escuela de Matemática 
Revista Universidad en Diálogo • Vol. 11, N. 2 2, Julio-Diciembre, 2021 • 31-43

ISSN 2215-2849 • EISSN: 2215-4752

URL: http://www.revistas.una.ac.cr/index.php/dialogo/index CORREO ELECTRÓNICO: universidadendialogo@una.cr DOI: http://doi.org/10.15359/udre.11-2.2

de la UNA, que manifiestan la falta de conocimientos y destrezas matemáticas que poseen al salir de la secundaria (Castillo, Gamboa e Hidalgo, 2017).

En relación con lo mencionado, se observa que los resultados en dicho examen en el año 2017 son similares a los obtenidos en años anteriores. Al respecto, en el año 2016, 1832 estudiantes realizaron el examen de diagnóstico y menos del $2,05 \%$ obtuvo una nota superior a 60 ; el $76,8 \%$ obtuvo calificaciones entre 20 y 40 y el promedio de las calificaciones obtenidas fue de 29,73 (Escuela de Matemática, 2017).

La realidad de la Región Brunca no se aleja de los resultados a nivel nacional, ya que es posible ver cómo a nivel de sus tres Direcciones Regionales de Educación los resultados no son alentadores. Según datos del Ministerio de Educación Pública, para los años del 2016-2018, el rendimiento en la Prueba de Bachillerato de Matemática no supera el $60 \%$, mientras que en las Direcciones Regionales de Grande de Térraba y Coto no supera el $52 \%$ de aprobación.

En lo que respecta a la situación de la educación universitaria y en particular el caso de la sede Región Brunca de la UNA, por ser la universidad pública que tiene mayor impacto en la región al tener dos Campus Universitarios, Pérez Zeledón y Coto, la situación muestra una problemática relacionada con los conocimientos matemáticos previos que poseen los estudiantes al ingresar a las carreras universitarias.

Al respecto, la Escuela de Matemática en su informe sobre el examen de diagnóstico de Matemática para estudiantes de primer ingreso en el año 2017, señala que en el Campus Pérez Zeledón el 94,74\% de los que aplicaron el examen tiene una nota inferior a 60, mientras que en el Campus Coto el porcentaje corresponde a un $98,25 \%$. Por su parte, la experiencia docente de los autores hace notar que los porcentajes de aprobación en los cursos introductorios de Matemática, para las carreras que se imparten en ambos campus no alcanzan en muchos casos ni el $35 \%$.

Todo lo anterior ejemplifica que los resultados que ha tenido la enseñanza de la Matemática en el país a nivel de educación primaria, secundaria y universitaria no son muy alentadores. Esta situación debe convertirse en un motivo de preocupación para las entidades encargadas de la formación de profesionales cuyas carreras se encuentren estrechamente ligadas con el área científica y tecnológica.

Es por ello que la formación, capacitación y actualización en el área de la matemática debe ser un trabajo orientado hacia los y las docentes en ejercicio. 
Por ejemplo, Meza y Agüero (2014) señalan que "los resultados obtenidos sugieren la necesidad de reforzar los procesos formativos de profesores de matemática o de fortalecer la capacitación profesional" (p. 11).

Es importante mencionar que existe la necesidad de brindar capacitaciones para actualizar a la comunidad docente sobre las nuevas tendencias y sobre todo en cuanto a cómo dar ese primer paso en pro del cambio de la educación costarricense.

De igual forma, Arias (2014), en su trabajo sobre la actuación de las personas tutoras y su relación con el proceso de aprendizaje de los y las docentes de Matemáticas en un programa de formación, señala que el fortalecimiento de la profesión docente constituye uno de los ejes prioritarios de actuación de muchas organizaciones en el mundo.

Todas estas situaciones crean en la Sede Regional Brunca de la Universidad Nacional un sentido de responsabilidad con la población educativa que recibe, ya que debe convertirse en partícipe directa a la hora de establecer estrategias, acciones y proyectos que promuevan el desarrollo educativo y social de la zona.

Es así como la implementación y el desarrollo de Matem-SRB en las instituciones de educación secundaria de la Región Brunca constituyen un esfuerzo por mejorar el panorama de la educación matemática, sobre todo en lo que respecta al desarrollo profesional del profesor o de la profesora de esta disciplina y la formación de estudiantes que pronto ingresarán a la universidad, fomentando la motivación por la disciplina y transmitiendo valores y actitudes que se requieren para tener éxito en la educación superior.

Además, se espera contribuir al aumento del nivel de aprobación en los cursos de Matemática de las universidades para garantizar la formación de profesionales en la región. Pero a su vez muestra el compromiso que tienen la Universidad Nacional, la Sede Regional Brunca y la Escuela de Matemática con la búsqueda del desarrollo y de la capacitación de las poblaciones en las áreas que presentan mayores problemáticas, y sin duda alguna el tema educativo es uno de ellos, principalmente en el área de la matemática.

\section{Objetivos del proyecto}

- Contribuir al crecimiento profesional de los y las docentes de Matemática de las Direcciones Regionales de Educación de Pérez Zeledón, Grande de Térraba y Coto, mediante el desarrollo de procesos de capacitación 
- Fortalecer la formación matemática de los y las estudiantes del Ciclo Diversificado de las Direcciones Regionales de Educación de Pérez Zeledón, Grande de Térraba y Coto, mediante la oferta de cursos de Matemática con un nivel universitario

\section{Metodología}

La implementación de Matem-SRB en la Región Brunca tiene como fin lograr una participación activa de las instituciones públicas de educación secundaria de la zona, que está conformada, según la distribución establecida por el Ministerio de Educación Pública, por las Direcciones Regionales de Educación (DRE) de Pérez Zeledón, Grande de Térraba y Coto; dando prioridad a aquellas instituciones que se encuentren en las zonas de menor desarrollo de la región.

Ahora bien, el proyecto busca fortalecer la enseñanza y el aprendizaje de la Matemática desde dos ejes principales. En primera instancia, busca trabajar con profesorado activo del Ciclo Diversificado de las DRE de Pérez Zeledón, Grande de Térraba y Coto, brindando de manera gratuita capacitaciones y actualización de contenidos, estrategias didácticas y metodológicas con especialistas en temáticas de su interés, esto mediante cursos virtuales, talleres, charlas u otras actividades que se consideren pertinentes. Además, estas actividades les permiten a los y las docentes compartir conocimientos y experiencias que contribuyan a la realimentación de los procesos de enseñanza y aprendizaje.

Como un segundo eje, el proyecto Matem-SRB busca fortalecer la formación académica de los y las estudiantes del Ciclo Diversificado que se matricularon en los colegios públicos de las regiones educativas mencionadas, al ofrecerles la oportunidad de matricular cursos universitarios de Matemática durante su estancia como estudiantes de secundaria.

Con esto se pretende que los y las estudiantes adquieran una sólida formación matemática, que va más allá de la recibida bajo el estándar de la educación media costarricense, incentivando y fomentando conocimientos matemáticos de índole conceptual y práctica que les permitan prepararse de una mejor manera para hacerle frente a experiencias académicas en un futuro. Además, tienen la oportunidad de ir conociendo el contexto universitario y familiarizándose con la UNA, como la universidad necesaria.

En relación con los cursos o las modalidades que ofrece Matem-SRB, estos se denominan Matem-Precálculo décimo, Matem-Precálculo undécimo y Matem-Cálculo. A continuación, se describe cada uno de ellos: 
1) Matem-Precálculo décimo: Corresponde a un curso anual en el que pueden inscribirse los y las estudiantes de las instituciones participantes en el proyecto, que estén cursando décimo año del Ciclo Diversificado (dieciséis años). Los temas que se desarrollan corresponden a números reales, álgebra, funciones, geometría analítica, geometría y trigonometría.

2) Matem-Precálculo undécimo: Corresponde a un curso anual en el que pueden inscribirse los y las estudiantes de las instituciones participantes en el proyecto, que estén cursando undécimo año del Ciclo Diversificado (diecisiete años). Los temas que se desarrollan corresponden a los mismos del proyecto Matem-Precálculo décimo.

3) Matem-Cálculo: Corresponde a un curso anual en el que pueden inscribirse los y las estudiantes de las instituciones participantes en el proyecto, que hayan aprobado el curso Matem-Precálculo décimo o Matem-Precálculo undécimo (diecisiete años). Los temas que se desarrollan corresponden a límites y continuidad, derivadas, aplicaciones de las derivadas, integrales indefinidas e integrales definidas.

La evaluación de cada uno de los cursos se realiza mediante cuatro pruebas escritas, que serán confeccionadas y calificadas por el personal académico a cargo del proyecto. La aplicación de las mismas se realizará en los campus universitarios de la UNA en Pérez Zeledón y Coto. Para la aprobación de estos cursos los y las estudiantes deben obtener una nota igual o superior a 7, de 10 , en su promedio ponderado, situación que les permitirá al finalizar el curso obtener un certificado, que, mediante un convenio existente entre las Escuelas de Matemática de las universidades estatales, les permitirá la convalidación de los cursos equivalentes a los que brinda el proyecto, esto mediante el acuerdo FCEN-EM-MATEM-911-2012, fechado el 12 de setiembre del año 2012.

Es importante aclarar que los cursos que imparte Matem-SRB son gratuitos, así como los materiales didácticos básicos que son entregados a los y las estudiantes (libro de texto y material adicional). Estos cursos son impartidos por tutores y tutoras que son docentes de Matemática de los centros educativos participantes, que serán responsables de su grupo a cargo y del nexo entre la coordinación del proyecto y el estudiantado. Además, se encargan de realizar la inscripción del centro educativo y de los y las estudiantes, de velar por el cumplimiento de los contenidos en los plazos establecidos, de retirar las calificaciones obtenidas por el estudiantado a su cargo en las evaluaciones realizadas y, en los casos que corresponda, de realizar las consultas o apelaciones a los exámenes aplicados. 


\section{Resultados}

Actualmente, el proyecto Matem-SRB se encuentra en su segundo año de aplicación, por lo que se presentan los resultados alcanzados en su primer año.

Para el 2019 se inscribieron un total de 220 estudiantes y 14 docentes de Matemática provenientes de 13 colegios de la Región Brunca. Esto evidencia el impacto que tiene este proyecto en zonas alejadas del área metropolitana. La distribución según la DRE de estos estudiantes y docentes se puede observar en la Tabla 1. En esta se muestra que la mayoría de estudiantes proviene de la DRE de Grande de Térraba (46\%), seguida de la DRE de Pérez Zeledón (30 \%).

\section{Tabla 1}

Estudiantes y docentes del proyecto Matem-SRB, año 2019, según Dirección Regional de Educación

\begin{tabular}{lcccc}
\hline \multirow{2}{*}{ Dirección Regional } & \multicolumn{2}{c}{ Estudiantes } & \multicolumn{2}{c}{ Docentes } \\
\cline { 2 - 5 } & Cantidad & $\%$ & Cantidad & $\%$ \\
\hline Pérez Zeledón & 65 & 30 & 3 & 21 \\
Grande de Térraba & 102 & 46 & 6 & 43 \\
Coto & 53 & 24 & 5 & 36 \\
\hline Total & 220 & 100 & 14 & 100 \\
\hline
\end{tabular}

\section{Nota. Elaboración propia}

Otro aspecto que se debe recalcar es que en cada uno de los cursos ofrecidos por Matem-SRB se dio la participación de estudiantes, siendo el de Precálculo de décimo el que tuvo más cantidad de estudiantes. Esta situación obedece a que varias personas tutoras consideraban que era mejor trabajar con estudiantes de décimo año, por tener más tiempo para ayudar en su preparación. De igual forma, se debe aclarar que los y las estudiantes que cursan Cálculo corresponden a un grupo que aprobó el curso Precálculo en proyectos homólogos. Dicha distribución se puede observar en la Tabla 2. 
URL: http://www.revistas.una.ac.cr/index.php/dialogo/index

CORREO ELECTRÓNICO: universidadendialogo@una.cr

DOI: http://doi.org/10.15359/udre.11-2.2

\section{Tabla 2}

Estudiantes del proyecto Matem-SRB, año 2019, según modalidad

\begin{tabular}{|c|c|c|}
\hline Nivel & Cantidad & $\%$ \\
\hline Precálculo, décimo & 123 & 56 \\
\hline Precálculo, undécimo & 70 & 32 \\
\hline Cálculo & 27 & 12 \\
\hline Total & 220 & 100 \\
\hline
\end{tabular}

Nota. Elaboración propia

Es importante mencionar que a lo largo del año 2019 se presentaron una serie de inconvenientes dentro de los centros educativos, principalmente relacionados con huelgas realizadas por docentes y estudiantes a nivel nacional, que afectaron de forma indirecta el desarrollo adecuado de Matem-SRB en los colegios. Aunado a esto, lamentablemente, algunas personas estudiantes y docentes no mostraron un verdadero interés y compromiso con el proyecto, por lo cual desertaron.

A pesar de estas situaciones, Matem-SRB continuó fomentando e incentivando el desarrollo de la enseñanza de la Matemática en la región, centrando sus esfuerzos en trabajar con aquellas personas participantes que continuaron, logrando alcanzar una participación activa de 7 colegios de educación secundaria, con 110 estudiantes y 6 docentes de Matemática.

Es importante aclarar que las estadísticas que se presentan a continuación hacen referencia a ese grupo de personas participantes que se mantuvieron activas hasta finalizar la implementación del primer año del proyecto.

La distribución del estudiantado y del cuerpo docente se puede observar según la Dirección Regional de Educación (Tabla 3), la cual muestra una evidente deserción de los y las estudiantes de la DRE de Grande de Térraba, ya que al inicio del proyecto tenía el grupo más grande. Este hecho puede deberse a que corresponde a una región alejada y de difícil acceso. Además, los y las docentes de esta DRE se desmotivaron, debido a que tenían que recuperar muchas lecciones para sacar adelante al estudiantado inscrito en el proyecto. 
Revista Universidad en Diálogo • Vol. 11, N. 2 2, Julio-Diciembre, 2021 • 31-43

ISSN 2215-2849 • EISSN: 2215-4752

URL: http://www.revistas.una.ac.cr/index.php/dialogo/index CORREO ELECTRÓNICO: universidadendialogo@una.cr

DOI: http://doi.org/10.15359/udre.11-2.2

\section{Tabla 3}

Estudiantes y docentes del proyecto Matem-SRB, año 2019, según Dirección Regional de Educación

\begin{tabular}{lcccc}
\hline \multirow{2}{*}{ Dirección Regional } & \multicolumn{2}{c}{ Estudiantes } & Docentes & \\
\cline { 2 - 5 } & Cantidad & $\%$ & Cantidad & $\%$ \\
\hline Pérez Zeledón & 44 & 40 & 1 & 16,67 \\
Grande de Térraba & 6 & 5,45 & 2 & 33 \\
Coto & 60 & 54,55 & 3 & 50 \\
\hline \multicolumn{1}{c}{ Total } & 110 & 100 & 6 & 100 \\
\hline
\end{tabular}

Nota. Elaboración propia

Por otra parte, se mantuvo una distribución homogénea según el nivel educativo de las personas participantes. Esto se evidencia en la Tabla 4, donde el 45,45\% cursaba el décimo nivel (dieciséis años).

\section{Tabla 4}

Estudiantes del proyecto Matem-SRB, año 2019, según nivel

\begin{tabular}{ccc}
\hline Nivel & Cantidad & $\%$ \\
\hline Décimo & 50 & 45,45 \\
Undécimo & 60 & 54,55 \\
\hline Total & 110 & 100 \\
\hline
\end{tabular}

Nota. Elaboración propia

Por su parte, la Tabla 5 muestra la participación de los y las estudiantes según la modalidad que brinda el proyecto. Es importante aclarar que los y las estudiantes que cursan Cálculo corresponden a un grupo que aprobó el curso Precálculo en proyectos homólogos.

\section{Tabla 5}

Estudiantes del proyecto Matem-SRB, año 2019, según modalidad

\begin{tabular}{lcc}
\hline \multicolumn{1}{c}{ Modalidad } & Cantidad & $\%$ \\
\hline Precálculo, décimo & 50 & 45,45 \\
Precálculo, undécimo & 34 & 30,10 \\
Cálculo & 26 & 24,45 \\
\hline \multicolumn{1}{c}{ Total } & 110 & 100 \\
\hline
\end{tabular}

Nota. Elaboración propia 
Se debe ser consciente de que los cursos que brinda el proyecto son de carácter universitario, razón por la cual, aunque los y las estudiantes se comprometan a culminar el curso matriculado y realicen los esfuerzos académicos, los resultados en ocasiones no son los que se esperan. Por lo cual no necesariamente los y las estudiantes que realizaron todas las pruebas que aplica el proyecto lograron aprobar el curso. Los resultados con respecto a la aprobación de los cursos ofrecidos por el proyecto se muestran en la Tabla 6.

\section{Tabla 6}

Cantidad de estudiantes que aprobaron los cursos del proyecto Matem-SRB durante el año 2019, según modalidad

\begin{tabular}{lccc}
\hline \multicolumn{1}{c}{ Modalidad } & Total de estudiantes & Estudiantes aprobados/as & $\%$ \\
\hline Precálculo, décimo & 50 & 22 & 44 \\
Precálculo, undécimo & 34 & 13 & 38,24 \\
Cálculo & 26 & 22 & 84,62 \\
\hline
\end{tabular}

Nota. Elaboración propia

La tabla anterior muestra que la mayor aprobación se dio en el curso de Cálculo, seguido de Precálculo de décimo. Esto puede deberse a que los y las estudiantes de Cálculo ya tenían la experiencia de llevar cursos de este tipo, pues habían aprobado el curso de Precálculo ofrecido en proyectos homólogos. Es relevante hacer notar que el porcentaje de aprobación del curso de Precálculo, tanto de décimo como de undécimo nivel, es similar a la aprobación de los cursos homólogos impartidos en instituciones públicas de educación superior.

\section{Conclusiones}

La enseñanza y el aprendizaje de la Matemática presentan una problemática dentro de la educación general básica costarricense; situación que se ve aún más marcada en las zonas alejadas del Gran Área Metropolitana. Es así como el proyecto Matem-SRB se convierte en una alternativa que les brinda a los y las docentes de Matemática en servicio, de las Direcciones Regionales de Educación de Pérez Zeledón, Grande de Térraba y Coto, la oportunidad de acceder a capacitaciones relacionadas con contenidos o estrategias didácticas que colaboren a su labor educativa. 
Revista Universidad en Diálogo • Vol. 11, N. 2 2, Julio-Diciembre, 2021 • 31-43

ISSN 2215-2849 • EISSN: 2215-4752

URL: http://www.revistas.una.ac.cr/index.php/dialogo/index CorReo ElECTRÓNICO: universidadendialogo@una.cr DOI: http://doi.org/10.15359/udre.11-2.2

Además, le proporciona al estudiantado del Ciclo Diversificado de estas regiones experiencias de utilidad en su incorporación al ambiente universitario, lo que fomenta una sólida formación en conocimientos y destrezas matemáticas, mediante la participación en cursos universitarios de Matemática.

A su vez, promueve los ideales de la regionalización y la acción social que se fomentan dentro de las universidades públicas de Costa Rica, al capacitar a un grupo de docentes y favorecer el nivel de conocimientos matemáticos de estudiantes que se ubican en regiones vulnerables.

Es importante mencionar que durante el primer año de implementación del proyecto se presentó un nivel de deserción que no era el esperado, por lo que se tomará como un proceso de aprendizaje con miras al mejoramiento del mismo. Ante esto, tanto las personas coordinadoras como los y las participantes (docentes de secundaria y estudiantes) establecerán estrategias y mecanismos que permitan la permanencia del estudiantado, así como una mayor participación y compromiso por parte de docentes y estudiantes. En este sentido, para el año 2020 se propusieron algunas clases virtuales para los y las estudiantes participantes, con la intención de apoyar la labor de los tutores y las tutoras y disminuir la deserción.

Por otro lado, Matem-SRB en un proyecto pionero en el área y en la región, ya que, aunque actualmente las demás universidades estatales cuentan con programas similares, estos se realizan generalmente en el Gran Área Metropolitana, dejando por fuera a zonas y poblaciones del país a las que, por condiciones geográficas y socioeconómicas, se les dificulta la participación en este tipo de proyectos.

Lo anterior también puede verse como una iniciativa que pueden promover otros centros de educación superior o entre distintas unidades académicas para establecer planes o proyectos en distintas áreas educativas, de manera que el estudiantado de estas regiones se vea beneficiado.

Finalmente, se debe ser consciente de que el desarrollo del proyecto no viene a convertirse en la solución definitiva a los problemas de la enseñanza y del aprendizaje de la Matemática que se presentan en la Región Brunca, pero sí constituye un primer acercamiento en la búsqueda de acciones concretas que colaboren a incrementar el desarrollo y aprendizaje de esta asignatura, buscando a su vez que los y las estudiantes tengan una base sólida de conocimientos que les permita incrementar su rendimiento académico a nivel de secundaria y, posteriormente, a nivel universitario. 


\section{Referencias}

Arias, M. (2014). Actuación de los tutores y su relación con el proceso de aprendizaje de los profesores de matemática en un programa de formación. (Tesis doctoral). Universidad de Granada, Granada, España.

Castillo, M., Gamboa, R. e Hidalgo, R. (2017). Algunos errores en matemática que cometen los estudiantes que ingresan a la universidad. En Y. Morales-López, M. Picado, R. Gamboa, C. Martínez, M. Castillo y R. Hidalgo (eds.), Memorias del VI Encuentro Provincial de Educación Matemática, Costa Rica, 2017 (pp. 69-82). Heredia: Universidad Nacional. ISBN: 978-9968-9661- 5-3. DOI: http://dx.doi.org/10.15359/ epem.6.17

Escuela de Matemática. (2017). Informe de resultados examen de diagnóstico de matemática: estudiantes de primer ingreso del año 2017. Universidad Nacional. Documento no publicado.

Estado de la Nación (2014). Estado de la Ciencia, Tecnología e información. San José. Programa Estado de la Nación.

Estado de la Nación (2017). Sexto Informe Estado de la Educación. San José. Programa Estado de la Nación.

Estado de la Nación (2019). Estado de la Educación. San José. Programa Estado de la Nación.

Meza, L. y Agüero, E. (2014). Evaluación de los aprendizajes en la educación media: características técnicas de las pruebas escritas en matemática. Revista Digital Matemática, 14(2), 1-12.

Ministerio de Educación Pública. (2017). Informe Nacional. Resultados de las pruebas nacionales de la Educación Formal 2016. Bachillerato. Dirección de Gestión y Evaluación de la Calidad. San José, Costa Rica.

Ministerio de Educación Pública. (2018). Informe Nacional, Promoción de Bachillerato 2017. Dirección de Gestión y Evaluación de la Calidad. San José, Costa Rica.

Ministerio de Educación Pública. (2019). Informe Nacional, Promoción de Bachillerato 2018. Dirección de Gestión y Evaluación de la Calidad. San José, Costa Rica. 